\title{
Study of Serum Sialic Acid And Microalbuminuria in Type 2 Diabetes Mellitus: A Case Control Study in North Coastal Andhrapradesh
}

\author{
Chand Basha Sky ${ }^{1}$, Hari Krishna Kasi ${ }^{1}$ \\ ${ }^{I}$ Department Of Biochemistry, Andhra Medical College Andhra Pradesh, India
}

\begin{abstract}
:
Background: Complications of diabetes are due to the endothelial dysfunction. Levels of sialic acid which is a major constituent of endothelium are increased in diabetes mellitus. Estimation of these levels can be used a marker in the development of microvascular and macrovascular complications.

Methods: A group of 60 clinical cases and 40 controls are taken in the study. Subjects were divided into three groups consisting of healthy controls, diabetics with nephropathy and diabetics without nephropathy. Correlations studies were performed between various groups.

Results: There is a positive correlation between serum sialic acid and both serum creatinine and microalbuminuria in controls $(r=0.036 ; r=0.070)$.In group 2 cases there is a positive correlation between serum sialic acid and serum creatinine $(r=0.089)$. There is a positive correlation between serum sialic acid and urine microalbumin in group 2 cases $(r=0.184)$.In group 3 cases the study revealed that there is a positive correlation between serum sialic acid and serum creatinine and urine microalbumin values $(r=0.345, r=0.782)$ respectively. A positive correlation between serum sialic acid and urine microalbumin in controls $(r=0.070)$ and in cases both group $2(r=0.184)$ and group $3(r=0.782)$.

Conclusion: Circulating serum sialic acid is an early manifestation of diabetic renal disease and hence sialic acid can be used as a marker of renal dysfunction in type 2 DM patients
\end{abstract}

Keywords: Sialic acid, Microalbuminuria, Nephropathy.

\section{Introduction}

Diabetes mellitus is a metabolic disorder of multiple etiologies characterized by chronic hyperglycemia with disturbances of carbohydrate, fat and protein metabolism resulting from defects in insulin secretion, insulin action or both. The prevalence of diabetes mellitus is continuously rising in both developed and developing countries which has reached epidemic proportion and has become one of the most challenging health problems of the 21 st century. The global prevalence of diabetes is expected to increase from $4 \%$ in 1995 to $5.4 \%$ by the year 2025. The total number of people with diabetes is projected to rise from 171 million in 2000 to 366 million in 2030.Currently the countries with the largest number of diabetic patients are India, China and United States.4 In India alone, diabetes is expected to increase from 40.6 million in 2006 to 79.4 million by 2030.In Indian diabetics most of the long standing micro and macro vascular complications are more common compared to other ethnic groups.6 Long term vascular complications represent the main cause of morbidity and mortality in diabetic patients. Diabetes mellitus is the leading cause of end stage renal disease (ESRD) and is responsible for $30-40 \%$ of all ESRD. Although type 1 and type 2 DM lead to ESRD, the great majority of patients are those with type $2 \mathrm{DM}$.

Serum sialic acid is a newly established potential risk factor for the development of micro and macrovascular complications of diabetes. 8 Serum sialic acid is a component of glycoprotein such as acute phase proteins and several serum acute phase proteins are elevated in diabetes. Serum sialic acid level is increased in both type 1 and type 2 diabetes mellitus patients with albuminuria. The possible mechanism associated with the role of sialic acid is in maintaining the negative charge of renal glomerular basement membrane which is one of the main regulators of membrane permeability. Due to increased vascular permeability there is shedding of vascular endothelial sialic acid into circulation.9

Microalbuminuria is the earliest manifestation of diabetic nephropathy and predictor of incipient nephropathy in diabetic patients. Early phase of diabetic renal disease called incipient diabetic nephropathy is characterized by increased albumin excretion in the range of $30-300 \mathrm{mg} / \mathrm{l}$. Urine is negative for standard dipsticks at this stage. Incipient diabetic nephropathy is reversible with tight glycemic control and ACE inhibitors. Once overt nephropathy is present, progression cannot be halted, only slowed. It is more effective to screen for early nephropathy with sensitive tests for microalbuminuria and to prevent the earliest stages of damage by vigorous control of hyperglycemia and hypertension. Hence the study was undertaken to estimate 
serum sialic acid and microalbuminuria and to correlate serum sialic acid and microalbuminuria in type $2 \mathrm{DM}$ patients.

\section{Materials and methods}

30 patients with clinically diagnosed type 2 diabetes mellitus with normoalbuminuria (group 2) and 30 patients with type 2 diabetes mellitus with microalbuminuria (group 3) are taken irrespective of age and sex who attended King George Hospital were included in the study. Age and sex matched healthy individuals were taken as control group(Group 1). Each gave informed consent. The patients and the controls voluntarily participated in this study.

\section{Inclusion criteria:}

For group 1 controls with normoglycemia.are taken. For group 2 and group 3 patients with diabetes having FBS $\geq 126 \mathrm{mg} / \mathrm{dl}$ are included.group 2 patients included those with microalbuminuria while that of group3 did not have any microalbunuria.

\section{Exclusion criteria:}

Patients suffering from acute and chronic inflammatory conditions, other metabolic conditions like ketoacidosis, cerebrovascular accidents, preeclamptic patients, pre-existing chronic kidney disease, chronic renal failure, chronic glomerulonephritis, nephrotic syndrome, smokers, alcoholics, patients with psychiatric disorders and primary hypertensives were excluded from study.

\section{Results}

The age distribution pattern of controls and cases under study ranges from 41 years to 80 years with mean age of $58.33 \pm 8.8$ in controls and $60.3 \pm 10.38$ in cases. Samples are gender matched with $56.66 \%$ of males and $43.33 \%$ of females in cases and $56.66 \%$ males and $43.33 \%$ females in controls. There is a positive correlation between serum sialic acid and both serum creatinine and microalbuminuria in controls $(\mathrm{r}=0.036$; $\mathrm{r}=0.070$ ).In group 2 cases there is a positive correlation between serum sialic acid and serum creatinine $(\mathrm{r}=0.089)$. There is a positive correlation between serum sialic acid and urine microalbumin in group 2 cases $(\mathrm{r}=0.184)$.In group 3 cases the study revealed that there is a positive correlation between serum sialic acid and serum creatinine and urine microalbumin values $(r=0.345, r=0.782)$ respectively.A positive correlation between serum sialic acid and urine microalbumin in controls $(r=0.070)$ and in cases both group $2(r=0.184)$ and group $3(\mathrm{r}=0.782)$.

\section{Discussion}

Type 2 diabetes mellitus is being increasingly recognized as a disease, which is characterised by dysfunction of the endothelium. The clinical markers of the generalized endothelial dysfunction becomes manifest in several forms.Microalbuminuria marks the onset of endothelial dysfunction related to the kidney. Microalbuminuria serves as a warning for imminent nephropathy. In present study we took 30 cases of diabetic mellitus with normoalbuminuria (group 2) and 30 cases of diabetes mellitus with microalbuminuria(group 3) who attended KGH and the study was compared with 30 healthy controls(group 1). In both cases and controls FBS, PPBS, serum creatinine, serum sialic acid and urinary microalbumin levels were measured.

Sialic acid acts as a cofactor of many cell surface receptors and positively associated with most of the serum acute phase reactants.Sialic acid can be used as a marker of acute phase response as many proteins of immune response are actually glycoproteins which have sialic acid as the terminal sugar on their oligosaccharide chain.Serum sialic acid concentration is elevated in pathological states when there is damage to tissue, tissue proliferation and inflammation. Sialic acid regulates vascular permeability. The vascular endothelium carries a high concentration of sialic acid hence extensive microvascular damage associated with NIDDM result in its shedding into the circulation. This leads to an increase in vascular permeability and increased serum sialic acid concentration. Tissue injury caused by diabetic vascular complications stimulates local cytokine secretions from cells involved in the complications such as macrophages and endothelium. This induces an acute phase response which involves the release of acute phase glycoproteins with sialic acid from the liver into the general circulation again leading to increased serum sialic acid concentrations.

In this study the mean serum sialic acid values were $1.87 \pm 0.31$ in controls and $2.18 \pm 0.27,3.04 \pm 0.35$ in group 2 and group 3 cases which is statistically highly significant $(\mathrm{P}<0.001)$. Serum sialic acid values were higher than controls when compared to group 2 and group 3 cases which correlated well with the clinical diagnosis.In a study done by Shivananda Nayak B, Geetha Bhaktha serum sialic acid levels were significantly increased in diabetics with severity of nephropathy and with the degree of urinary albumin excretion In another study, done by Shahid SM and Mahaboob T, it was shown that serum sialic acid levels were significantly increased in both diabetic and diabetic nephropathy patients as compared to controls. This elevation was 
significantly higher in diabetic nephropathy patients compared to diabetic without nephropathy. Crook $\mathrm{M}$ et.al also found that serum sialic acid was significantly higher in patients with diabetic complications than in those without complications. This study is in accordance with the study done by Chen JW, Gall MA, increase in serum sialic acid was demonstrated with an increasing urinary albumin excretion rate in NIDDM patients with normoalbuminuria,and microalbuminuria patients compared to controls. 100

\section{Urinary Microalbumin}

Microalbuminuria is an important risk factor for cardiovascular disease and progressive renal impairment. Microalbuminuria arises from the increased passage of albumin through the glomerular filtration barrier which results from ultrastructural changes rather than alterations in glomerular pressure or filtration rate alone.Microalbuminuria predicts the development of overt diabetic nephropathy in type 1 and type 2 DM but the relationship is less clear in type 2 because of heterogeneity and presence of other risk factor for microalbuminuria in these elderly patients.In this study the mean values of urinary microalbumin were $8.86 \pm 2.40$ in controls and $16 \pm 6.08,128.6 \pm 67.29$ in group 2 and group 3 cases which is statistically highly significant $(\mathrm{P}<0.001)$. Urinary microalbumin values were higher than controls when compared to cases which correlated well with the clinical diagnosis.

\section{Correlation Between Serum Sialic Acid And Other Study Parameters.}

Correlation study revealed a small positive correlation between serum sialic acid and both FBS and PPBS in type 2 diabetic mellitus cases with normoalbuminuria $(r=0.217 ; \mathrm{r}=0.147)$,and positive correlation between serum sialic acid and both FBS and PPBS in type 2 diabetes patients with microalbuminuria $(\mathrm{r}=0.319 ; \mathrm{r}=0.575)$ when compared with controls indicating the role of hyperglycemia towards renal damage.This correlation is distorted in diabetic mellitus cases when compared to control groups as there is small negative correlation between serum sialic acid and PPBS in controls $(r=-0.085)$ but there is a small positive correlation between serum sialic acid and FBS $(\mathrm{r}=0.111)$ in control group. There is a positive correlation between serum sialic acid and serum creatinine in cases $(r=0.089 ; r=0.345)$ showing that as serum creatinine increases serum sialic acid also increases but this correlation is not distorted when cases were compared with controls as controls also showed trivial correlation $(\mathrm{r}=0.036)$. The correlation study revealed very large positive correlation between serum sialic acid and urinary microalbumin $(r=0.782)$ in type 2 diabetes mellitus with microalbuminuria cases showing that as microalbumin excretion increases, serum sialic acid also increases pointing contributory role of serum sialic acid towards renal damage. There is a small positive correlation seen between serum sialic acid and microalbuminuria in type 2 diabetes with normoalbuminuria patients $(\mathrm{r}=0.184)$ This correlation is not distorted in cases when compared to controls as there is a very small positive correlation between serum sialic acid and urinary microalbumin $(r=0.070)$ in controls.

\section{Conclusion}

Various hypotheses have been proposed till date on how type 2 DM progresses in human subjects. These include the involvement of renin- angiotensin system, advanced gyration end product formation (AGE), endothelial dysfunction and oxidative stress, Our study was undertaken to study the serum sialic acid levels and microalbuminuria and to assess whether there is a relationship between these two in normoalbuminuric and microalbuminuric patients as compared to controls. In this study a very large positive correlation was observed between serum sialic acid and urinary microalbumin in group 3 cases( $(r=0.782)$, where as a small positive correlation in group 2 cases $(r=0.184)$. It was also observed that serum sialic acid concentrations were strongly associated with renal dysfunction ( creatinine,microalbuminuria) and the development of micro and macrovascular complications.

These markers were clinically correlated with increasing concentration of sialic acid. It is concluded that increase in circulating serum sialic acid is an early manifestation of diabetic renal disease and hence sialic acid can be used as a marker of renal dysfunction in type 2 DM patients

\section{References}

[1]. Nayak BS, Roberts L. Relationship between inflammatory markers, metabolic and anthropometric variables in the Caribbean type 2 diabetic patients with and without microvascular complications. Journal of Inflammation 2006, 3:17 doi:10.1186/1476-9255-3-17

[2]. Kumar R, Abbas A, Fausto N, Aster JC, editors. Robbins and cotron "Pathologic basis of disease" 8th Ed. Saunders Elsevier Publishing Division; 2010. 934-935, 1131-1146

[3]. Mogensen CE, Christensen CK. Predicting diabetic nephropathy in insulin dependent patients. N Engl J Med1984; $311: 364-72$.

[4]. Kasper DL, Braunwald E, Fauci AS, Hauser SL, Lango DL, Jameson JL et al, editors. Harrison's principles of internal medicine. 16th ed. McGraw-Hill: Medical publishing division; 2008

[5]. Castano L, Eisenbarth GS. Type-I diabetes. A chronic autoimmune disease of human, mouse and rat. Annual Review of Immunology 1990;8:647-79 